\title{
Single-Camera Computational Stereo Using a Rotating Mirror
}

\author{
A. F. Clark and S. W. Chan \\ Image Processing Group \\ Department of Electronic Systems Engineering \\ University of Essex \\ Wivenhoe Park, Colchester CO4 3SQ, UK \\ alien@essex.ac.uk
}

\begin{abstract}
The use of a single camera and rotating mirror for depth estimation in a periscope-like system is examined. It is found that such an arrangement is viable and that the problem of matching features in successive frames may be greatly simplified by comparatively straightforward pre-processing. Experimental results are presented and some possible applications of such a compact stereo sensor are outlined.
\end{abstract}

\section{Introduction}

Depth perception is one of the more important functions of the human visual system. Although several cues are involved - shading, relative size, etc. - stereo plays a crucial rôle. Computational stereo, now in widespread use in computer vision, naturally mimics the human visual system by having two cameras viewing the 3-D scene [2]. In recent years, single-camera approaches to computational stereo have become of interest: the simplest of these merely replace the two cameras with a mirror system [8] or glass plate [7], though more recent work has employed inclined mirrors [3]. Such arrangements suffer from two disadvantages: they have a limited field of view and, in order to produce an acceptable baseline, must be physically big.

There are a number of vision tasks in which the physical size of the sensor limits its usage: examples of this are the inspection of the insides of aircraft engines and medical endoscopy. In such cases, physical size and a panoramic field of view are both important factors. Both of these may be met by employing the principle of the periscope in computational stereo. This is the purpose of this paper "periscopic stereo."

The periscope has, of course, been used for viewing in awkward configurations for many, many years, though its potential for computational stereo does not appear to have been exploited in vision systems. Panoramic cameras, as found in remote sensing, and linescan imagers (used in military aircraft) have essentially the same imaging geometry [9]. A conical mirror, which also features a similar geometry, has been used for panoramic stereo [10], though two viewpoints were 


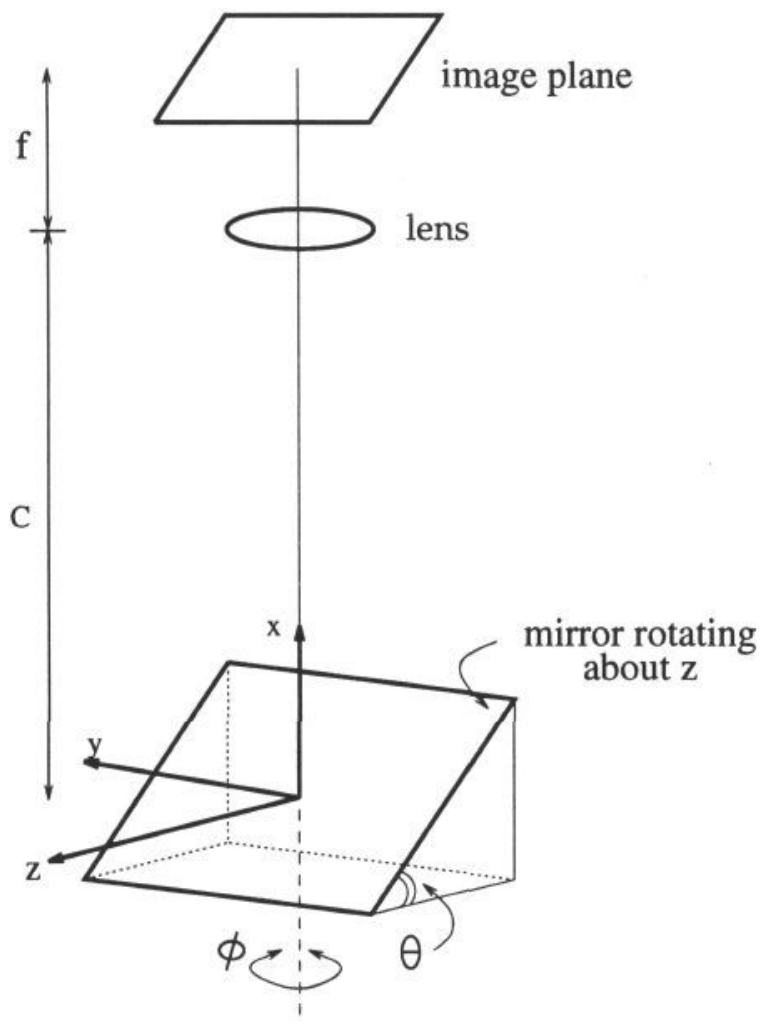

Figure 1: Schematic Diagram of Mirror Arrangement

still employed. The fact that rotation may be used as the basis of a stereo viewing geometry was first reported widely in [4]. Subsequent work [6] replaced the rotating camera with a rotating mirror, analyzing the case in which the axis of rotation of the mirror is perpendicular to the optical axis of the camera and reported simulation, though not experimental, results. The same paper also gave a preliminary analysis of the case considered here, in which the axis of rotation of the mirror lies along the optical axis (Figure 1).

This paper gives a detailed analysis of the periscopic geometry. It indicates how, with a suitably calibrated system, the problem may be reduced to an essentially one-dimensional one. Experimental results with a prototype system are presented and some potential applications are briefly considered.

\section{Camera Motion in Rotating Systems}

The basic arrangement of the system is shown in Figure 2. Light from the scene is reflected by a mirror positioned at the origin and inclined at an angle $\theta$ onto a camera whose lens is centred at the point 


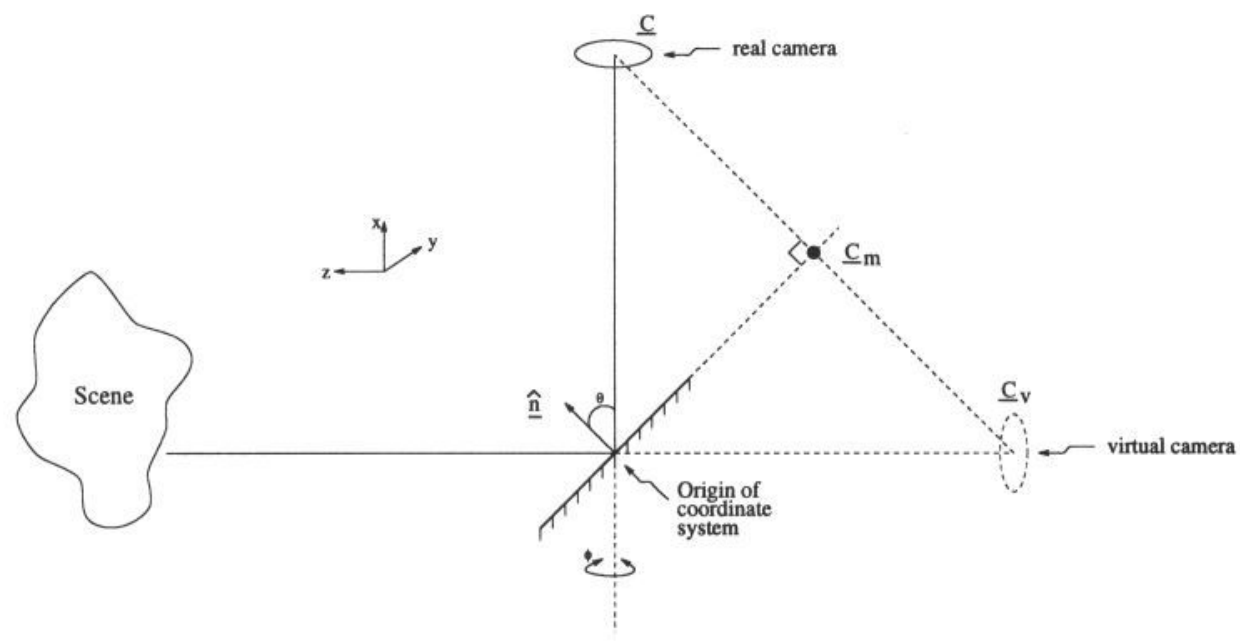

Figure 2: Side Elevation of Optical System

$$
\underline{C}=\left(\begin{array}{l}
C \\
0 \\
0
\end{array}\right)
$$

i.e., the camera is placed a distance $C$ away from the mirror along the $x$-axis. (The notation and the general approach to the analysis are similar to those of [6].) The mirror is made to rotate about the $x$-axis by an angle $\phi$. We proceed by considering a 'virtual' camera: this occupies a position equivalent to the real camera but 'behind' the mirror; i.e., it is the position at which the real camera would have to be located if the mirror were not present.

From Figure 2, we observe that the surface normal, $\underline{\hat{n}}$, is given by

$$
\left(\begin{array}{c}
\cos \theta \\
\sin \theta \sin \phi \\
\sin \theta \cos \phi
\end{array}\right) .
$$

In order to find the location of the virtual camera, $\underline{C}_{v}$, we first consider the perpendicular projection of the real camera position onto the mirror plane (see Figure 2):

$$
\underline{C}_{m}=\underline{C}-\alpha \underline{\hat{n}} .
$$

$\underline{C}_{m}$ is in the mirror plane, so $\underline{C}_{m} \cdot \underline{\hat{n}}=0$. Hence,

$$
(\underline{C}-\alpha \underline{\hat{n}}) \cdot \underline{\hat{n}}=0
$$

and consequently $\alpha=C \cos \theta$. The position of the virtual camera may be written as

$$
\underline{C}_{v}=\underline{C}-2 \alpha \underline{\hat{n}},
$$


from which one may calculate $\underline{C}_{v}$ :

$$
\underline{C}_{v}=-C\left(\begin{array}{c}
\cos 2 \theta \\
\sin 2 \theta \sin \phi \\
\sin 2 \theta \cos \phi
\end{array}\right) \text {. }
$$

As the mirror rotates, $\phi$ varies. The $x$-component of $\underline{C}_{v}$ is unaffected by such rotation, as we would expect, while the $y$ - and $z$-components undergo circular motion in the $x=0$ plane.

The above derivation allows us to determine the movement of the centre of the virtual camera as the mirror moves but it does not allow us to determine the movement of an arbitrary point on the image. To do that, let us consider a point

$$
\underline{C}^{\prime}=\left(\begin{array}{c}
C+\delta x \\
\delta y \\
\delta z
\end{array}\right)
$$

i.e., displaced by amounts $\delta x, \delta y$ and $\delta z$ from $\underline{C}$ along the $x$-, $y$-, and $z$-axes respectively. This will have projection

$$
\underline{C}_{m}^{\prime}=\underline{C}^{\prime}-\alpha^{\prime} \underline{\hat{n}}
$$

on the mirror plane, from which we obtain

$$
\alpha^{\prime}=\underline{C^{\prime}} \cdot \underline{\hat{n}} .
$$

The corresponding virtual position is

$$
\begin{aligned}
\underline{C}_{v}^{\prime} & =\underline{C}-2 \alpha^{\prime} \underline{\hat{n}} \\
& =\underline{C}^{\prime}-2\left(\underline{C^{\prime}} \cdot \underline{\hat{n}}\right) \underline{\hat{n}} .
\end{aligned}
$$

Now,

$$
\underline{C}^{\prime} \cdot \underline{\hat{n}}=\cos \theta(C+\delta x)+\sin \theta \sin \phi \delta y+\sin \theta \cos \phi \delta z
$$

and, substituting (11) into (10), we obtain

$$
\underline{C}_{v}^{\prime}=\left(\begin{array}{c}
-\cos 2 \theta(C+\delta x)-\sin 2 \theta \sin \phi \delta y-\sin 2 \theta \cos \phi \delta z \\
-\sin 2 \theta \sin \phi(C+\delta x)-\left(2 \sin ^{2} \theta \sin ^{2} \phi-1\right) \delta y-2 \sin ^{2} \theta \sin \phi \cos \phi \delta z \\
-\sin 2 \theta \cos \phi(C+\delta x)-2 \sin ^{2} \theta \sin \phi \cos \phi \delta y-\left(2 \sin ^{2} \theta \cos ^{2} \phi-1\right) \delta z
\end{array}\right)
$$

which simplifies to (6) when $\delta x=\delta y=\delta z=0$.

A comparison of (6) and (12) shows that the motion of a point that does not lie on the optical axis consists of two components: the circular motion of the virtual camera around the origin and a rotation of $\underline{C}_{v}^{\prime}$ around the optical axis. This realization allows the motion of images recorded by the camera system to be decomposed into a more useful form, as detailed in the next section. 


\section{Decomposition of Rotations}

We may write (6) and (12) in the form

$$
\begin{array}{r}
\underline{C}_{v}=\underline{T} \cdot \underline{C} \\
\underline{C}_{v}^{\prime}=\underline{T} \cdot \underline{C^{\prime}}
\end{array}
$$

respectively for some transformation $\underline{T}$ which maps from real to virtual coordinates. The difference between these positions is

$$
\underline{C}_{v}^{\prime}-\underline{C}_{v}=\underline{T} \cdot\left(\underline{C}^{\prime}-\underline{C}\right)
$$

from which we obtain

$$
\underline{T}=-\left(\begin{array}{ccc}
\cos 2 \theta & \sin 2 \theta \sin \phi & \sin 2 \theta \cos \phi \\
\sin 2 \theta \sin \phi & 2 \sin ^{2} \theta \sin ^{2} \phi-1 & 2 \sin ^{2} \theta \sin \phi \cos \phi \\
\sin 2 \theta \sin \phi & 2 \sin ^{2} \theta \sin \phi \cos \phi & 2 \sin ^{2} \theta \cos ^{2} \phi-1
\end{array}\right) .
$$

This expression for $\underline{T}$ must contain the two rotational components mentioned above in addition to any other transformations due to the imaging process. In particular, it must contain a rotation through an angle $\phi$ about the $x$-axis, $\underline{R}_{\phi}$, and a modification of the axes' directions due to the mirror. Taking both rotation and reflection into account, we may determine $\underline{T}^{\prime}$, the transformation without these effects; from $\underline{T}^{\prime}=\underline{R}_{\phi} \underline{T}$, where

$$
\underline{R}_{\phi}=\left(\begin{array}{ccc}
1 & 0 & 0 \\
0 & \cos \phi & -\sin \phi \\
0 & \sin \phi & \cos \phi
\end{array}\right)
$$

assuming a right-handed coordinate system. Performing the matrix multiplication, we obtain

$$
\underline{T}^{\prime}=-\left(\begin{array}{ccc}
\cos 2 \theta & \sin 2 \theta \sin \phi & \sin 2 \theta \cos \phi \\
0 & -\cos \phi & \sin \phi \\
\sin 2 \theta & -\cos 2 \theta \sin \phi & -\cos 2 \theta \cos \phi
\end{array}\right) .
$$

This expression shows that, after removing the effect of the rotation of a point $\underline{C}_{v}^{\prime}$ around $\underline{C}_{v}$, there is still a transformation present that depends upon $\phi$ : in effect, the imaging system has been reduced to a planar configuration.

The effect of this simple rotation of the image thus changes a difficult stereo matching problem, one which would require the matching of image features that are affected by translation and rotation between frames, to a much simpler one in which the feature matches lie along scan lines. The following section describes how one may estimate the distance to points in the far field from measurements obtained from these rotation-compensated images. 


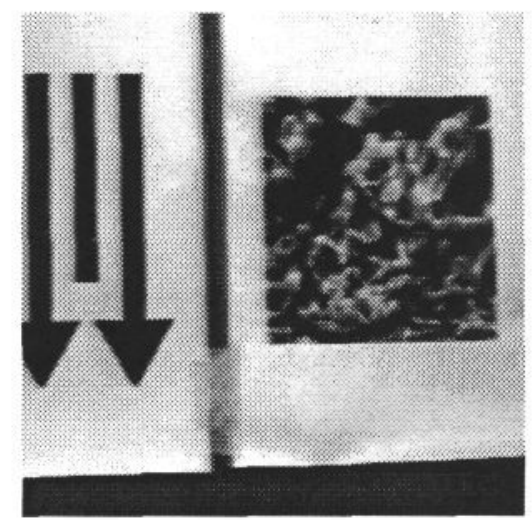

(a) Original image no. 535

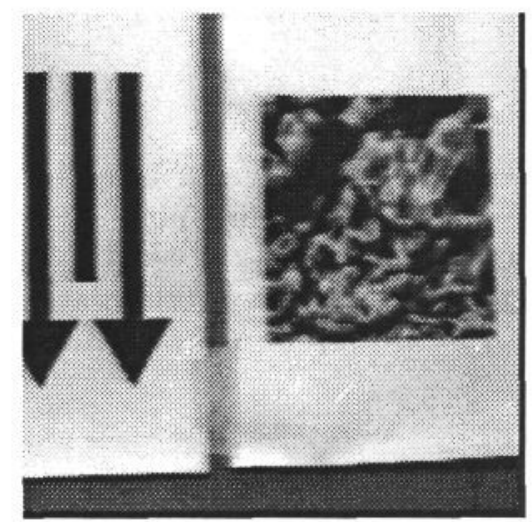

(c) Rotation-compensated image no. 535

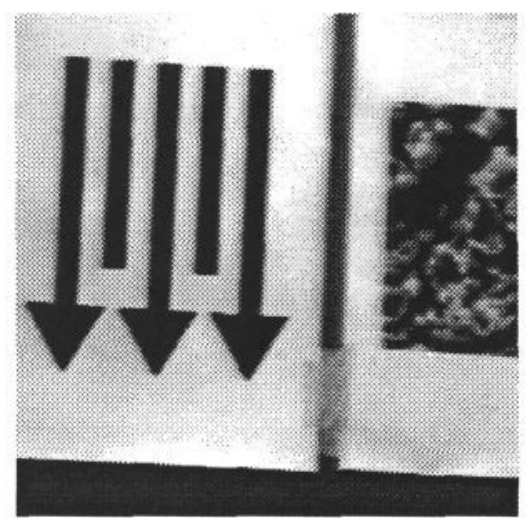

(b) Original image no. 534

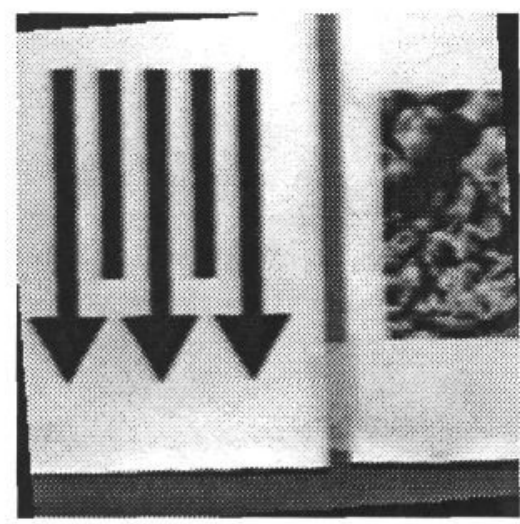

(d) Rotation-compensated image no. 534

Figure 3: The Rotation Compensation Process

\section{Depth Estimation from Rotation-Compensated Images}

Some images recorded from a system of the type of Figure 1 are shown in Figure 3 along with their rotation-compensated versions. Close inspection of these images will show that disparities may be observed: this means that one may use the images to estimate the distance from the observer using an approach similar to that for conventional computational stereo. Indeed, it is not difficult to derive equations that permit depth to be estimated from these rotation-compensated images. In doing so, we shall make the usual assumption that the camera may be accurately described by a 'pin-hole' model.

Let us consider a point

$$
\underline{P}=\left(\begin{array}{l}
X \\
Y \\
Z
\end{array}\right)
$$


in the scene that is imaged to $\underline{P}_{r}$ in the back focal plane of the real camera. Let us write

$$
\underline{P}_{r}=\left(\begin{array}{l}
x \\
y \\
z
\end{array}\right)
$$

By similar triangles, we have

$$
m_{x}=\frac{x}{f}=\frac{Y}{X-C} .
$$

Applying the transformation $\underline{T}^{\prime}$ from real to virtual coordinates yields

$$
m_{x}=\frac{-\cos \phi Y+\sin \phi Z}{\cos 2 \theta X+\sin 2 \theta \sin \phi Y+\sin 2 \theta \cos \phi Z+C} .
$$

In the case when $\theta=\pi / 4,(16)$ may be simplified to

$$
m_{x}=\frac{-\cos \phi Y+\sin \phi Z}{\sin \phi Y+\cos \phi Z+C}
$$

which may be re-arranged to give

$$
x \equiv C m_{x}=\left(\begin{array}{ll}
-m_{x} \sin \phi-\cos \phi & \sin \phi-m_{x} \cos \phi
\end{array}\right)\left(\begin{array}{c}
Y \\
Z
\end{array}\right)
$$

This equation corresponds to (13) of [6], except that $2 \phi \rightarrow \phi$. If one considers the differences between imaging geometries here and in [6], one will see that (17) can be obtained from that in [6] by a purely physical argument.

Now, (17) gives an equation in two unknowns ( $Y$ and $Z$ ) that relates to a single image measurement, $x$. However, we are free to vary $\phi$ : doing so builds up multiple 'instances' of (17) that refer to the same $Y$ and $Z$, and we may then use numerical techniques to produce estimates of $Y$ and $Z$.

\section{Experimental Results}

The authors have constructed a non-real-time demonstrator using components that one would expect to find in any image processing laboratory. This system uses a plane mirror of $\sim 10 \times 10 \mathrm{~cm}$ which is rotated at $3.84^{\circ} /$ frame by a turntable ${ }^{1}$ : these allow a reasonable overlap between consecutive frames; indeed, there is still an overlap between every second frame. A video camera is aligned such that its optical axis (i.e., the centre of captured images) is aligned with the centre of rotation of the turntable. Images are captured in real time but processed in non-real-time.

An important experimental consideration concerns the camera. The frame rate of a video camera is sufficiently slow that the rotation of the turntable will cause motion blurring. The authors therefore utilized a shuttered CCD video camera.

\footnotetext{
${ }^{1}$ This is 16 revolutions/minute, which should give the astute reader an idea of the equipment employed.
} 
Even with a shuttered camera, rotational blurring is apparent between the fields of a frame: only one field of each frame is used to circumvent this.

In our initial experiments, it has not been feasible to monitor the rotation of the turntable directly. The manufacturer's rating of the turntable motor gives nominal values for the rotation speed and its error. However, experimental measurement of this important quantity is also possible. This is achieved by capturing a sequence of frames covering a complete revolution of the turntable. The first frame captured is then correlated with frames around the expected best match and the correlation values interpolated to yield an accurate figure for the number of frames captured in one revolution. (The timing error of a video camera is of the order of $1 \mathrm{in} 10^{6}$ at worst, so this is negligible.) Hence, the mean angle between frames is easily calculated; its error is sufficiently small that it could not cause the feature match to be shifted by as much as a scan line.

The demonstrator system was set up in a corner of a laboratory with several objects placed at known distances around it. A sequence of images was then captured and processed to estimate the distance to these objects. The approach adopted here is a simple one, and there are undoubtedly better ones: the authors are more concerned with proving the principle of periscopic stereo than with general stereo matching.

The captured images were initially sub-sampled and rotated about the rotation origin on the image using bilinear interplation. The rotation-compensated images were passed through an interest operator to identify distinct features. The Moravec [5] operator was used for simplity: this attempts to select good features over small windows by returning areas that have local maxima of a directional variance measure, calculated by finding the sums of squares of differences of pixels adjacent in each of four directions over each window.

Each feature found in the first frame was extracted and used as a template for the second image. A correlation tracker [1] was used to locate the feature in the next image. The accuracy of feature location was enhanced by interpolating correlation values around the neighbourhood of the peak; it is estimated that the resulting accuracy is about \pm 0.5 pixels. The located features were then inserted into (17) and least-square optimization used to estimate values for $Y$ and $Z$.

These experimental results, comparing the distances calculated using periscopic stereo and directly-measured values, are shown graphically in Figure 4. Ideally, the points should lie on the line of unit gradient: taking errors into account, these results are close to this ideal. The errors indicated assume a 0.5-pixel error in the location of features in each frame, giving a one-pixel error in the disparity. They do not include other factors (e.g., error in the measurement of $C$ or the focal length) and are therefore under-estimates of the probable errors.

\section{Conclusions and Further Work}

This paper has investigated 'periscopic stereo' and found that, although the problem initially appears to be a very difficult one, a simple decomposition of the rotations present greatly simplifies it. This suggests a procedure for producing stereo images, both for direct viewing and for the estimation of depth. Our experimental measurements have proven the viability of the technique. 


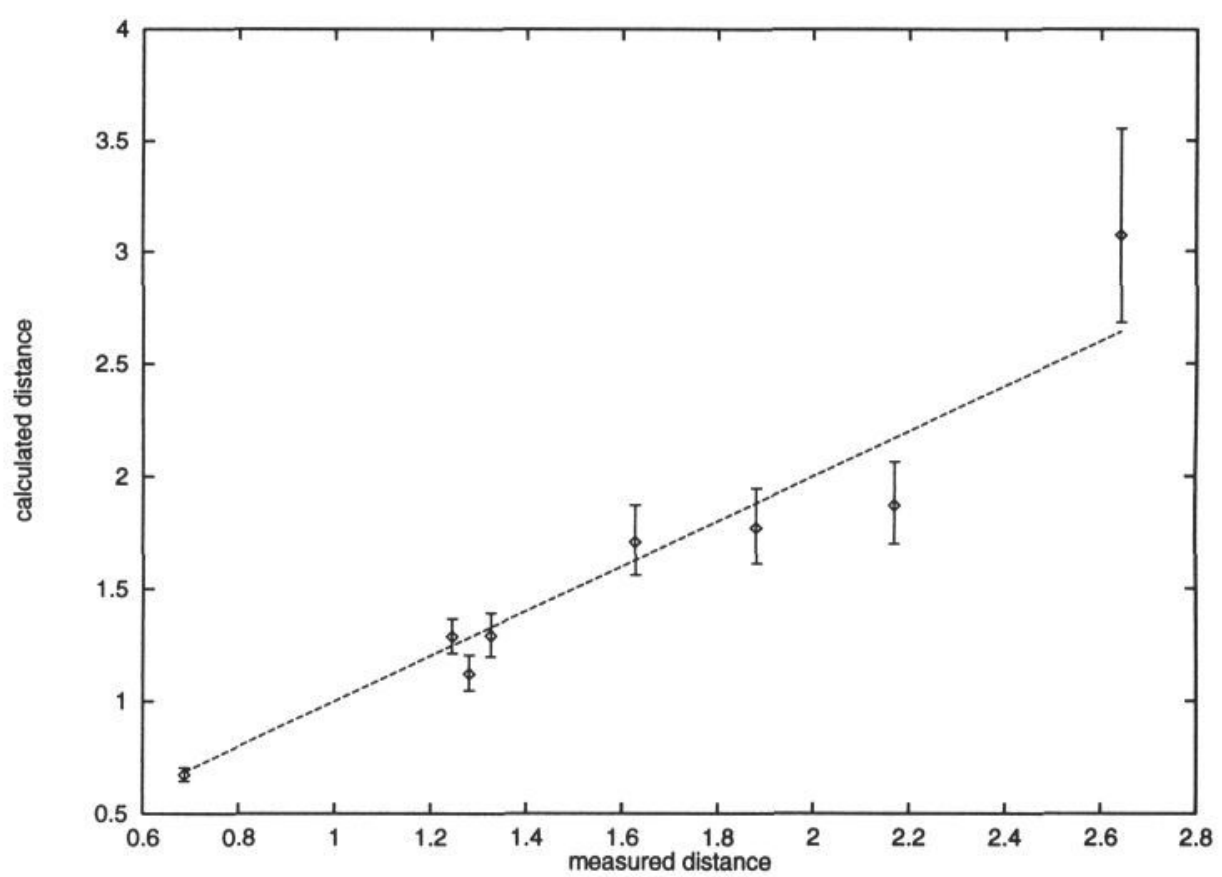

Figure 4: Comparison of Directly-Measured and Calculated Distances

This technique may be used to provide a panoramic distance measurement capability for, say, mobile robots. However, with careful design, it will be possible to make the sensor very compact. Periscopic stereo may thus be regarded as being complementary to, rather than a competitor to, conventional stereo: the former is well suited to viewing the outside of objects, while periscopic stereo may well find a niche in exploring the inside of objects such as aircraft engines; it may also be able to provide a compact stereoscope and distance estimation system for endoscopy, which could be greatly beneficial for medical techniques such as keyhole surgery.

Although the principle has been proven, further work is undoubtedly required before the technique becomes mature. A major topic is undoubtedly calibration: the parameters for which conventional stereo systems are calibrated are not necessarily those for which a periscopic stereo system requires calibration. Work is well progressed into this problem. Finally, there is a need to demonstrate that the system is capable of real-time operation: construction of suitable hardware is under way.

\section{Acknowledgements}

Parts of this work were carried out in association with project IED3/1/2171 Parallel Reconfigurable Image Processing Systems. 


\section{References}

[1] A. F. Clark and M. Köküer. Feature identification and model tracking for model-based coding. In Proceedings of the 11th Int. Conf. Patt. Rec., volume III, pages 79-82, The Hague, The Netherlands, August 1992.

[2] U. R. Dhond and J. K. Aggarwal. Structure from stereo. IEEE Transactions on Systems, Man and Cybernetics, 19(6):1489-1510, November 1989.

[3] A. Goshtasby and W. A. Gruver. Design of a single-lens stereo camera system. Pattern Recognition, 26(6):923-936, 1993.

[4] H. Ishiguro, M. Yamamoto, and S. Tsuji. Omni-directional stereo. IEEE Transactions on Pattern Analysis and Machine Intelligence, 14(2):257-262, February 1992.

[5] H. P. Moravec. Rover visual obstacle avoidance. In Proceedings of the 7 $\frac{\text { th }}{\text { h }}$ International Conference on Artificial Intelligence, pages 785-790, University of British Columbia, Vancouver, Canada, August 1981.

[6] D. W. Murray and P. A. Beardsley. Range recovery using multi-camera stereo. In D. Hogg and R. Boyle, editors, British Machine Vision Conference 1992. Springer-Verlag, September 1992.

[7] Y. Nishimoto and Y. Shirai. A feature-based stereo model using small disparities. In Proceedings of the International Conference on Computer Vision and Pattern Recognition, pages 192-196, 1987.

[8] W. Teoh and X. D. Zhang. An inexpensive stereoscopic vision system for robots. In Proceedings of the International Conference on Robotics, pages 186-189, 1984.

[9] P. R. Wolf. Elements of Photogrammetry. McGraw-Hill, 1983.

[10] Y. Yagi and S. Kawato. Panoramic scene analysis with conic projection. In IEEE International Workshop on Intelligent Robots and Systems, pages 181190, Washington DC, USA, 1990. IEEE Computer Society Press. 